\title{
Filocrono de genótipos de arroz irrigado em função de época de semeadura
}

\author{
Phyllochron of rice genotypes as a function of sowing date
}

\author{
Nereu Augusto Streck ${ }^{\mathrm{I}}$ Simone Michelon ${ }^{\mathrm{II}}$ Hamilton Telles Rosa ${ }^{\mathrm{III}}$ Lidiane Cristine Walter ${ }^{\mathrm{III}}$ \\ Leosane Cristina Bosco ${ }^{\text {III }}$ Gizelli Moiano de Paula ${ }^{\text {III }}$ Cátia Camera ${ }^{\text {III }}$ \\ Flávia Kaufmann Samboranha ${ }^{\text {III }}$ Elio Marcolin ${ }^{\text {IV }}$ \\ Sidinei José Lopes ${ }^{\mathrm{I}}$
}

\section{RESUMO}

O filocrono é definido como o tempo necessário para o aparecimento de folhas sucessivas em um colmo, no caso de espécies da família das Poáceas. Através do filocrono, pode-se calcular o número de folhas acumuladas, o que é uma excelente maneira de caracterizar o desenvolvimento vegetal. Este trabalho teve por objetivo estimar o filocrono de sete cultivares de arroz irrigado utilizadas na Região Sul do Brasil, em cinco épocas de semeadura, na região ecoclimática da Depressão Central do Rio Grande do Sul. Um experimento de campo foi realizado em Santa Maria, RS, durante os anos agrícolas de 2003/2004 e 2004/2005, com cinco épocas de semeadura em cada ano agrícola. Foram utilizadas sete cultivares de arroz recomendadas para a Região Sul do País: "IRGA 421”, "IRGA 416”, "IRGA 417”, "IRGA 420”, “BRIRGA 409”, “BRS 7 TAIM” $e$ "EPAGRI 109”. O filocrono $\left({ }^{\circ} \mathrm{C}\right.$ dia folha $^{-1}$ ) foi estimado pelo inverso do coeficiente angular da regressão linear entre o número de folhas no colmo principal, determinado conforme o Estádio de Haun, e a soma térmica diária acumulada (temperatura base $=11^{\circ} \mathrm{C}$ ) a partir da emergência das plantas. As diferenças genotípicas são pequenas $e$ podem ser desprezadas, enquanto que o ambiente, principalmente pela época de semeadura, tem grande efeito sobre o filocrono em arroz.

Palavras-chave: Oryza sativa L., aparecimento de folhas, desenvolvimento vegetal, temperatura.

\section{ABSTRACT}

The phyllochron is defined as the time needed for appearance of successive leaves on a culm in plants of the Poaceae family. Using the concept of phyllochron, it is possible to calculate the number of accumulated leaves, which is an excellent parameter to measure the plant development. The objective of this study was to estimate the phyllochron of seven rice cultivars grown in Southern Brazil in five sowing dates in the Central Region of Rio Grande do Sul State. A two-year field experiment was carried out in Santa Maria, RS, Brazil during 2003/2004 and 2004/2005 growing seasons, with five sowing dates each year. Seven rice cultivars recommended for Southern Brazil were used: IRGA 421, IRGA 416, IRGA 417, IRGA 420, BR-IRGA 409, BRS 7 TAIM and EPAGRI 109. The phyllochron $\left({ }^{\circ} \mathrm{C}\right.$ day leaf $\left.{ }^{1}\right)$ was estimated by the inverse of the slope of the linear regression between main culm number of accumulated leaves (represented by the Haun Stage) and thermal time (base temperature $=11^{\circ} \mathrm{C}$ ) accumulated from plant emergence. Genotype differences are small and can be neglected, but the environment, mainly through sowing date, has a large effect on phyllochron in rice.

Key words: Oryza sativa L., leaf appearance, plant development, temperature.

\section{INTRODUÇÃO}

O arroz (Oryza sativa L.) é uma das mais importantes culturas agrícolas no Brasil. É o terceiro cereal mais produzido e consumido no mundo, ocupando cerca de 150 milhões de hectares (USDA, 2005), além de fazer parte da dieta básica de dois terços da população do planeta (LUZZARDI et al., 2005; FERREIRA et al., 2005). Ao longo da história, o arroz

\footnotetext{
IDepartamento de Fitotecnia, Centro de Ciências Rurais (CCR), Universidade Federal de Santa Maria (UFSM). Avenida Roraima, 1000, 97105-900, Santa Maria, RS, Brasil. E-mail: nstreck1@smail.ufsm.br. Autor para correspondência.

IIPrograma de Pós-graduação em Agronomia, CCR, UFSM.

${ }^{\text {IIIC } C u r s o ~ d e ~ G r a d u a c ̧ a ̃ o ~ e m ~ A g r o n o m i a, ~ C C R, ~ U F S M . ~}$

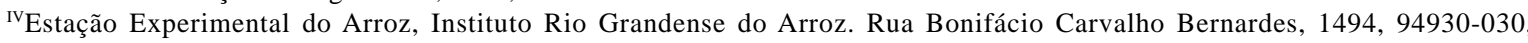
Cachoeirinha, RS.
} 
tem sido um dos alimentos mais importantes na nutrição humana, com melhor balanceamento nutricional e de maior potencial de aumento de produção para combate da fome no mundo. Portanto, a pesquisa com essa cultura pode afetar o bem-estar de um considerável número de pessoas.

Caracterizar o desenvolvimento de genótipos de arroz em diferentes épocas de semeadura é importante para avaliar sua adaptação e sua recomendação e para o manejo da cultura. O número de folhas acumuladas no colmo principal (NF) é uma excelente maneira de caracterizar o desenvolvimento vegetal. A iniciação, a diferenciação e o aparecimento de muitos órgãos em espécies da família das Poáceas, como o arroz, estão relacionados com o NF, como, o início e o fim da diferenciação da panícula (McMASTER et al., 1991; ELLIS et al., 1993; CALDERINI et al., 1996; LEE et al., 2001; STRECK et al., 2003), a emissão de perfilhos (KLEPPER et al., 1983; McMASTER et al., 1991; RICKMAN \& KLEPPER, 1991; TIVET et al., 2001; JAFFUEL \& DAUZAT, 2005), o emborrachamento e o aparecimento da estrutura reprodutiva (ROBERTSON et al., 1996; JAMIESON et al., 1998; LEE et al., 2001; STRECK et al., 2003). Além disso, o aparecimento de folhas é importante na evolução da área folhar da planta, a qual está relacionada com a interceptação da radiação solar pelo dossel vegetal, com a fotossíntese, com a acumulação de fitomassa e com o rendimento de grãos da cultura (McMASTER et al., 1991; TIVET et al., 2001).

Uma maneira de estimar o NF é através do conceito de filocrono, que varia um pouco conforme alguns autores. KLEPPER et al. (1982) definiram o filocrono como sendo o intervalo de tempo entre a emissão de duas folhas sucessivas em poáceas. Por sua vez, WILHELM \& McMASTER (1995) conceituaram o filocrono como o intervalo de tempo entre estádios similares de desenvolvimento de folhas, enquanto RICKMAN \& KLEPPER (1995) definem o filocrono como sendo o intervalo de tempo entre o aparecimento de folhas sucessivas em uma haste ou colmo. A unidade de filocrono, portanto, é tempo de aparecimento de uma folha (tempo folha-1).

Uma unidade freqüentemente usada para contabilizar tempo em plantas são os dias do calendário civil (exemplo: dia do ano, dias após a semeadura ou a emergência). A unidade de tempo em dias, no entanto, não é a mais adequada, pois o desenvolvimento da planta de arroz é muito influenciado pela temperatura do ar (OLDEMAN et al., 1986; ELLIS et al., 1993; SIÉ et al., 1998). Portanto, uma unidade realística do tempo biológico em plantas deve incluir esse elemento meteorológico.

Uma maneira de incluir a temperatura do ar no tempo vegetal é utilizar a soma térmica (com unidade
${ }^{\circ} \mathrm{C}$ dia) acima de uma temperatura mínima ou base, a qual tem sido demonstrada como uma melhor medida de tempo biológico em plantas do que os dias do calendário civil(GILMORE \& ROGERS, 1958; RUSSELE et al., 1984; McMASTER \& SMIKA, 1988). O filocrono, nesse caso, é o intervalo, em graus-dia, entre a emissão de folhas sucessivas, com unidade ${ }^{\circ} \mathrm{C}$ dia folha ${ }^{-1}$ (HERMES et al., 2001; XUE et al., 2004).

Existem muitas cultivares de arroz disponíveis e recomendadas para cultivo nas várzeas dos estados do Rio Grande do Sul e de Santa Catarina, sendo anualmente lançados novos genótipos. A caracterização do desenvolvimento desses genótipos em diferentes agroecossistemas, especialmente das épocas de semeadura, é um tema relevante e contínuo de pesquisa que pode contribuir para melhorar a eficiência da cadeia produtiva do arroz.

O presente trabalho teve por objetivo estimar o filocrono de sete cultivares de arroz irrigado utilizadas na Região Sul do Brasil, em cinco épocas de semeadura, na região ecoclimática da Depressão Central do Rio Grande do Sul.

\section{MATERIAL E MÉTODOS}

Um experimento de campo foi realizado na área experimental do Departamento de Fitotecnia da Universidade Federal de Santa Maria, Santa Maria, RS (latitude de $29^{\circ} 43^{\prime}$ S; longitude de $53^{\circ} 43^{\prime}$ W W e altitude de 95 m), durante dois anos agrícolas (2003/2004 e 2004/ 2005). O clima local, segundo a classificação climática de Köppen, pertence ao tipo Cfa, que significa subtropical úmido, com verões quentes e sem estação seca definida (MORENO, 1961). O solo do local é uma transição entre a unidade de mapeamento São Pedro (Argissolo Vermelho distrófico arênico) e a unidade de mapeamento Santa Maria (Alissolo Hipocrômico argilúvico típico) (EMBRAPA, 1999).

Foram implantadas cinco épocas de semeadura em cada ano agrícola. No ano agrícola 2003/ 2004, as datas de semeadura foram: 01/09/2003, 20/10/ 2003, 21/11/2003, 05/01/2004 e 29/01/2004, e, no ano agrícola 2004/2005, as datas de semeadura foram: 02/ 09/2004, 07/10/2004, 04/11/2004, 03/12/2004 e 02/03/ 2005. As épocas de semeadura foram escolhidas para se ter plantas desenvolvendo antes, durante, e após a época recomendada para a cultura, que é de final de outubro a meados de dezembro (SOSBAI, 2005), e assim, serem expostas a diferentes condições de temperatura do ar. A última data de semeadura no segundo ano agrícola foi mais tarde comparado com o primeiro ano agrícola, a fim de submeter as plantas a temperaturas ainda mais baixas do que no ano anterior. 
Foram utilizadas sete cultivares de arroz irrigado com ciclos de desenvolvimento distintos: "IRGA 421" (muito precoce), "IRGA 416” (precoce), "IRGA 417” (precoce), “IRGA 420” (médio), “BR-IRGA 409” (médiotardio), "BRS 7 TAIM” (médio-tardio) e "EPAGRI 109” (tardio). Essas cultivares são da subespécie índica e com tipo de planta moderno (estatura baixa) e recomendadas para cultivo na Região Sul do país (SOSBAI, 2005).

A análise dos dados foi realizada segundo um esquema trifatorial $(5 \times 2 \times 7)$ : cinco épocas de semeadura, dois anos agrícolas e sete cultivares de arroz, no delineamento inteiramente casualizado, com quatro repetições, cada uma composta de um balde com 10 plantas.

As plantas de arroz foram cultivadas em baldes com capacidade de 12 litros, preenchidos com solo do local, mantidos com a borda $5 \mathrm{~cm}$ acima do nível da superfície do solo. O espaçamento entre os baldes (distâncias entre os centros) foi de 1,5m x 0,8m.

Foram distribuídas 30 sementes por balde e cobertas com uma camada de aproximadamente $3 \mathrm{~cm}$ de solo. O dia da emergência foi considerado quando 50\% das plântulas estavam visíveis acima do solo. No estágio V3 (COUNCE et al., 2000), fez-se um raleio, deixando-se 10 plântulas por balde (o que resultou em uma densidade de aproximadamente 200 plantas $\mathrm{m}^{-2}$ ), das quais cinco foram identificadas, aleatoriamente, com arames coloridos. Nesse momento, também se iniciou a irrigação para manter uma lâmina constante de água de $5 \mathrm{~cm}$ até o final do ciclo das plantas. A irrigação foi feita diariamente com água de torneira, manualmente com mangueiras plásticas. Durante os meses de dezembro, janeiro e fevereiro, freqüentemente eram necessárias duas irrigações por dia. O manejo da cultura (adubações e tratamentos fitossanitários) seguiu as recomendações técnicas para a cultura do arroz irrigado no Sul do Brasil (SOSBAI, 2005).

No colmo principal dessas plantas marcadas, foram medidos, uma vez por semana, o comprimento da última e da penúltima folha e contado o número de folhas, até que as plantas tivessem 13 folhas. Com esses dados, foi calculado o estádio de Haun (HS; HAUN, 1973) no colmo principal, que representa o número de folhas completamente expandidas (NF) mais a razão entre os comprimentos da última ( $\left.\mathrm{L}_{n}\right)$ e da penúltima ( $\left.\mathrm{L}_{\mathrm{n}-1}\right)$ folhas, através da equação (HAUN, 1973):

$\mathrm{HS}=(\mathrm{NF}-1)+\mathrm{L}_{\mathrm{n}} / \mathrm{L}_{\mathrm{n}-1} \quad\{$ folhas $\} \quad(1)$

As temperaturas mínima e máxima diárias do ar a $1,5 \mathrm{~m}$ da superfície do solo gramado, durante os dois períodos experimentais, foram medidas em uma estação meteorológica convencional pertencente ao 8o Distrito de Meteorologia/Instituto Nacional de Meteorologia (DISME/INMET) e localizada a aproximadamente $200 \mathrm{~m}$ da área experimental. A soma térmica diária (STd) foi calculada conforme proposto por GILMORE \& ROGERS (1958) e ARNOLD (1960) através da equação:

$$
\mathrm{STd}=(\mathrm{Tm}-\mathrm{Tb}) .1 \mathrm{dia} \quad\left\{{ }^{\circ} \mathrm{C} \text { dia }\right\} \quad(2)
$$

Onde: Tmé a temperatura média diária do ar, calculada pela média aritmética das temperaturas mínima e máxima diárias do ar, e Tb é a temperatura base para o arroz, definida como a temperatura mínima abaixo da qual não há emissão de folhas. Assumiu-se a $\mathrm{Tb}=11^{\circ} \mathrm{C}$ (INFELD et al., 1998). A soma térmica diária foi acumulada a partir da emergência, resultando na soma térmica acumulada (STa), ou seja:

$$
\text { STa }=\Sigma \text { STd } \quad\left\{{ }^{\circ} \mathrm{C} \text { dia }\right\} \quad \text { (3) }
$$

Foi realizada uma regressão linear entre HS no colmo principal e STa para cada repetição (média das cinco plantas identificadas por balde). O filocrono foi estimado como sendo o inverso do coeficiente angular da regressão linear (KLEPPER et al, 1982; KIRBY, 1995; XUE et al., 2004). As médias de filocrono de cada repetição foram submetidas a análise de variância para avaliar o efeito de fontes de variação (cultivar, época de semeadura e ano agrícola). Foi calculada a diferença mínima significativa (DMS) a 5\% de probabilidade de erro. A análise da variância foi feita com a média das cinco plantas por balde.

\section{RESULTADOS E DISCUSSÃO}

Valores elevados de correlação entre HS e STa foram verificados para todas as cultivares nos dois anos, com coeficiente de determinação $\left(r^{2}\right)$ acima de 0,93 para todas as épocas de semeadura. Um exemplo da relação entre HS e STa está representado na figura 1. A alta relação linear indica que a temperatura do ar é o principal elemento meteorológico que governa o aparecimento de folhas em arroz, e que a estimativa do filocrono pelo método da regressão linear entre HS e STa é uma metodologia apropriada (XUE et al., 2004; STRECK et al., 2005).

A análise de variância da variável filocrono mostrou efeito significativo dos três fatores principais (cultivar, época de semeadura e ano agrícola), das interações duplas dos fatores e da interação tríplice (Tabela 1). Entre os fatores principais, o maior quadrado médio foi o da época de semeadura, o que sugere que a variação do filocrono é mais afetada pelo ambiente do que pela constituição genética da variedade de arroz. O maior valor do quadrado médio da interação dupla ano agrícola x época de semeadura (Tabela 1) confirma essa tendência. 


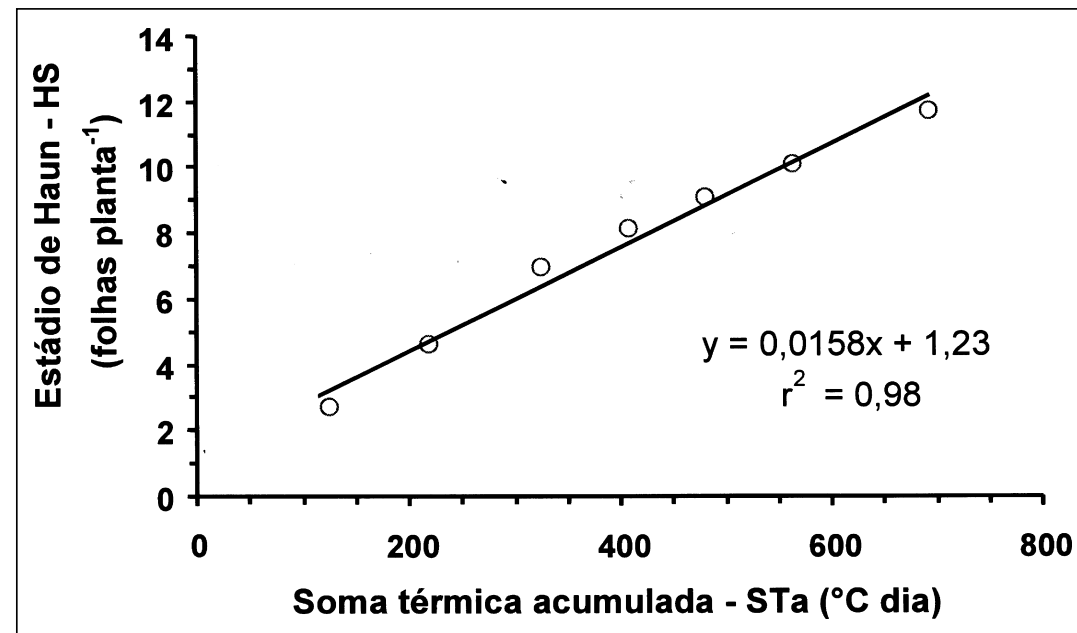

Figura 1 - Exemplo da metodologia usada na estimativa do filocrono em arroz irrigado. Os valores correspondem a uma das repetições (balde com cinco plantas) da cV. "IRGA 420", do experimento de 2004/2005, na semeadura em 03/12/ 2004. O filocrono estimado neste caso é $63,3^{\circ} \mathrm{C}$ dia folha ${ }^{-1}$. Santa Maria, RS.

Portanto, para fins práticos, pode-se desconsiderar a diferença de filocrono entre os sete genótipos estudados. Em trigo, diversos autores obtiveram filocrono similar entre genótipos (KLEPPER et al., 1982; McMASTER et al., 1992; FRANK \& BAUER, 1995; XUE et al., 2004). Entretanto, a diferença de filocrono entre épocas de semeadura foi acentuada, especialmente no ano agrícola 2004/2005 (Figura 2). Nesse ano, por exemplo, a diferença média de filocrono das cultivares entre as épocas 1 (02/09/04) e 5 (02/03/05) foi de $22,4^{\circ} \mathrm{C}$ dia folha ${ }^{-1}$. Assumindose 12 folhas no colmo

As cultivares apresentaram valores de filocrono diferentes estatisticamente entre si, a 5\% de probabilidade de erro, pois o DMS para cultivar foi de $1,3^{\circ} \mathrm{C}$ dia folha ${ }^{-1}$. Valores de filocrono para cultivares foram diferentes nos dois anos (Figura 2), pois o DMS para ano foi de $0,7^{\circ} \mathrm{C}$ dia folha ${ }^{-1}$. No entanto, analisandose os valores de filocrono das cultivares (Figura 2) e o do quadrado médio do fator principal cultivar (Tabela 1), biologicamente, o valor de DMS de $1,3^{\circ} \mathrm{C}$ dia folha ${ }^{-1}$ é pequeno. Por exemplo, se a planta de arroz produz 12 folhas no colmo principal, como foi o caso de todas as cultivares neste estudo, uma diferença de $1,3^{\circ} \mathrm{C}$ dia folha $^{-1}$ causaria uma diferença de $16,6^{\circ} \mathrm{C}$ dia até o aparecimento da folha bandeira, o que corresponde a aproximadamente apenas um dia do calendário civil.

Tabela 1 - Graus de liberdade (GL) e quadrado médio (QM) do quadro de análise da variância para a variável filocrono de sete cultivares de arroz irrigado, semeadas em cinco épocas, em dois anos agrícolas (2003/2004 e 2004/2005). Santa Maria, RS.

\begin{tabular}{lcc}
\hline Fonte & GL & QM \\
\hline Cultivar & 6 & $155,11^{* *}$ \\
Época de semeadura & 4 & $1083,1^{* *}$ \\
Ano agrícola & 1 & $85,80^{* *}$ \\
Cultivar*época de semeadura & 24 & $25,19 * *$ \\
Ano agrícola*cultivar & 6 & $47,94^{* *}$ \\
Ano agrícola*época de semeadura & 4 & $1268,14^{* *}$ \\
Ano agrícola*cultivar*época de semeadura & 24 & $37,21^{* *}$ \\
$* *$ Probabilidade $<0,0001$ & & \\
\hline
\end{tabular}

principal, a diferença de soma térmica para o surgimento da folha bandeira nesse caso é de $268,8^{\circ} \mathrm{C}$ dia, o que corresponde a muitos dias do calendário civil.

No experimento de 2003/2004, o filocrono variou de $49,6^{\circ} \mathrm{C}$ dia folha ${ }^{-1}$ para a cultivar "IRGA 421", na época $1(01 / 09 / 2003)$, a 66,3 ${ }^{\circ} \mathrm{C}$ dia folha ${ }^{-1}$ para a cultivar "IRGA 420", na época 2 (20/10/2003) (Figura 2). Em geral, a cultivar "IRGA 421" teve menor valor de filocrono, exceto na época 5 (29/01/2004), quando a cultivar "IRGA 416 " apresentou filocrono de $51,1^{\circ} \mathrm{C}$ dia folha ${ }^{-1}$ (valor mais baixo para esta época). O maior valor foi observado na cultivar "IRGA 420", nas épocas 1, 2 e $3(21 / 11 / 2003)$.

Em relação às épocas de semeadura no ano agrícola 2003/2004, é possível afirmar-se que a emissão de folhas é mais lenta (filocrono maior) nas épocas que estão dentro do período recomendado para a Região da Depressão Central do Estado do Rio Grande do Sul (SOSBAI, 2005) que, no experimento, estão representadas na Região pelas épocas 2 e 3 . Nas semeaduras realizadas fora da época recomendada, a emissão de folhas é acelerada, apresentando menores valores de filocrono.

No experimento realizado em 2004/2005, o menor valor de filocrono foi $42,9^{\circ} \mathrm{C}$ dia folha ${ }^{-1}$ na cultivar “IRGA 420", na época 1 (02/09/2004), e o maior valor foi $77,7^{\circ} \mathrm{C}$ dia folha ${ }^{-1}$ na cultivar “EPAGRI 109 ”, na época 5 (02/03/2005) (Figura 2). Nesse ano, as datas de semeadura foram diferentes das do primeiro ano, principalmente na época $4(03 / 12 / 2004)$, que foi adiantada em aproximadamente um mês, e na época 5 , que foi atrasada em mais de um mês. Aépoca 4, portanto, 


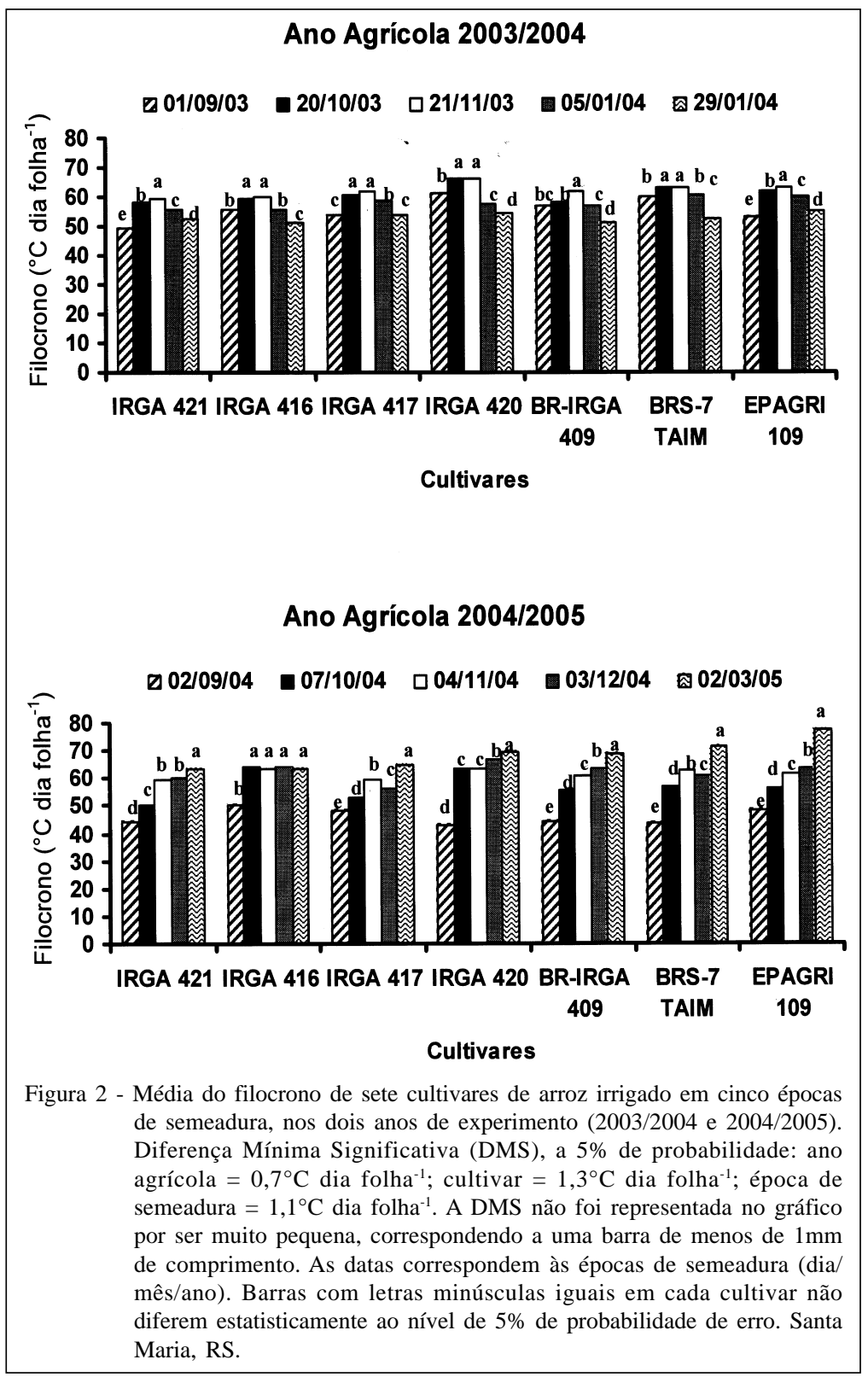

filocrono, Figura 2), uma possível hipótese para esse resultado é alguma tolerância maior dessa cultivar às baixas temperaturas do que as das outras cultivares, hipótese que ainda precisa ser confirmada em trabalhos posteriores.

Diferentes valores de filocrono (emissão de folhas) em trigo e de plastocrono (emissão de nós) em melão entre épocas de semeadura ao longo do ano também são relatados na literatura (McMASTER \& WILHELM, 1995; KIRBY, 1995; STRECK et al., 2005). É possível que o uso de soma térmica como medida de tempo no filocrono seja uma das causas principais de variação do filocrono entre épocas de semeadura (STRECK et al., 2005). Apesar da simplicidade de cálculo e da soma térmica ser uma medida de tempo biológico mais realística do que os dias do calendário civil (GILMORE \& ROGERS, 1958; RUSSELE et al., 1984; McMASTER \& SMIKA, 1988), o método está aberto a críticas por ter a pressuposição de haver uma relação linear entre desenvolvimento e temperatura do ar (XUE et al., 2004). Essa pressuposição é adequada apenas para uma faixa de temperatura, entre as temperaturas base e ótima de desenvolvimento. Próximo a essas temperaturas cardinais, a resposta do desenvolvimento vegetal à temperatura do ar, incluindo a emissão de folhas, é não-linear (SHAYKEWICH, 1995; STRECK et al., 2003; STRECK, 2004). Quando o ciclo da cultura ocorre na época recomendada para seu cultivo, geralmente as temperaturas se situam na faixa de resposta linear de emissão de folhas à temperatura do ar. No entanto, quando o cultivo é realizado fora da época recomendada, como foi o caso de três épocas de semeadura nos dois anos agrícolas desse estudo, as temperaturas se situam com menos freqüência na região de resposta linear a esse elemento meteorológico, o que pode ter causado diferenças grandes de filocrono das cultivares de arroz entre épocas de semeadura e entre anos. 


\section{CONCLUSÕES}

O ambiente em que as plantas se desenvolvem afeta mais o filocrono do que o genótipo em arroz irrigado. Diferenças genotípicas de filocrono podem ser desprezadas, mas a época de semeadura tem grande efeito sobre o filocrono em arroz. A pressuposição de linearidade no cálculo de soma térmica, a unidade de tempo do filocrono, é apontada como a principal causa de variação do filocrono de arroz entre épocas de semeadura.

\section{AGRADECIMENTOS}

À Coordenação de Aperfeiçoamento de Pessoal de Nível Superior (CAPES), pela concessão de bolsa de mestrado à Michelon. Ao Fundo de Incentivo a Pesquisa (FIPE) da UFSM, pela concessão de bolsa de Iniciação Científica (BIC) à Walter. Ao Programa Institucional de Bolsas de Iniciação Científica do Conselho de Desenvolvimento Científico e Tecnológico (PIBIC/ CNPq/ UFSM), pela concessão de bolsa ao pesquisador Bosco e pela bolsa de Produtividade em Pesquisa concedida à Streck.

\section{REFERÊNCIAS}

ARNOLD, C.Y. Maximum-minimum temperatures as a basis for computing heat units. Proceedings of the American Society for Horticultural Sciences, v.76, p.682-692, 1960.

CALDERINI, D.F. et al. Appearance and growth of individual leaves as affected by semidwarfism in isogenic lines of wheat. Annals of Botany, v.77, p.583-589, 1996.

COUNCE, P. et al. A uniform, objective, and adaptive system for expressing rice development. Crop Science, v.40, n.2, p.436-443, 2000.

ELLIS, R.H. et al. Rates of leaf appearance and panicle development in rice (Oryza sativa L.): a comparison at three temperatures. Agricultural and Forest Meteorology, v.66, p.129-138, 1993.

EMBRAPA. Sistema Brasileiro de Classificação de Solos. Brasília: Embrapa produções de informações, 1999. 412p.

FERREIRA, C.M. et al. Qualidade do arroz no Brasil: evolução e padronização. Santo Antônio de Goiás: Embrapa Arroz e Feijão, 2005. 61p.

FRANK, A.B.; BAUER, A. Phyllochron differences in wheat, barley and forrage grasses. Crop Science, v.35, n.1, p.19-23, 1995.

GILMORE, E.C.; ROGERS, J.S. Heat units as a method of measuring maturity in corn. Agronomy Journal, v.50, n.10, p.611-615, 1958.

HAUN, J.R. Visual quantification of wheat development. Agronomy Journal, v.65, p.116-119, 1973.

HERMES, C.C. et al. Emissão de folhas de alface em função da soma térmica. Revista Brasileira de Agrometeorologia, v.9, n.2, p.269-275, 2001.
INFELD, A.J. et al. Temperatura-base e graus-dia durante o período vegetativo de três grupos de cultivares de arroz irrigado. Revista Brasileira de Agrometeorologia, v.6, n.2, p.187191, 1998.

JAFFUEL, S.; DAUZAT, J. Synchronism of leaf and tiller emergence relative to position and to main stem development stage in a rice cultivar. Annals of Botany, v.95 p.401-412, 2005 .

JAMIESON, P.D. et al. Making sense of wheat development: a critique of methodology. Field Crops Research, v.55, p.117$127,1998$.

KIRBY, E.J.M. Environmental factors influencing the phyllochron. Crop Science, v.35, n.1, p.11-19, 1995.

KLEPPER, B. et al. Leaf and tiller identification on wheat plants. Crop Science, v.23, p.1002-1004, 1983.

KLEPPER. B. et al. Quantitative characterization of vegetative development in small cereal grains. Agronomy Journal, v.74, n.5, p.789-792, 1982.

LEE, C.K. et al. Heading date and final leaf number as affected by sowing date and prediction of heading based on leaf appearance model in rice. Korean Journal of Crop Science, v.46, n.3, p.195-201, 2001.

LUZZARDI, R. et al. Avaliação preliminar da produtividade em campo e qualidade industrial de híbridos de arroz no Rio Grande do Sul. In: CONGRESSO BRASILEIRO DE ARROZ IRRIGADO, 4., 2005, Santa Maria, RS. Anais... Santa Maria: Sociedade SulBrasileira de Arroz Irrigado, 2005. V.1. 567p. p.70-72.

MORENO, J.A. Clima do Rio Grande do Sul. Porto Alegre: Secretaria de Agricultura, Diretoria de Terras e Colonização, Secção de Geografia, 1961. 43p.

McMASTER, G.S.; SMIKA, D.E. Estimation and evolution of winter wheat phenology in the Central Great Plains. Agricultural and Forest Meterology, v.43, n.1, p.1-18, 1988.

McMASTER, G.S. et al. Simulation of shoot vegetative development and growth of unstressed winter wheat. Ecological Modelling, v.53, p.189-204, 1991.

McMASTER, G.S. et al. Simulating winter wheat shoot apex phenology. Journal of Agricultural Science, v.119, n.1, p.1-12, 1992.

McMASTER, G.S.; WILHELM, W.W. Accuracy of equation predicting the phyllochron of wheat. Crop Science, v.35, n.1, p.30-36, 1995.

OLDEMAN, L.R. et al. Response of rice to weather variables. In: OLDEMAN, L.R. International workshop on the impact of weather parameters on growth and yield of rice. Los Baños: IRRI, 1986. p.5-39.

RICKMAN, R.W.; KLEPPER, B.L. Tillering in wheat. In: HODGES, T. Predicting crop phenology. Boston: CRC, 1991. p.73-83.

RICKMAN, R.W.; KLEPPER, B.L. The phyllochron: where do we go in the future? Crop Science, v.35, n.1, p.44-49, 1995. 
ROBERTSON, M.J. et al. Temperature response to vernalization in wheat: modelling the effect on the final number of main stem leaves. Annals of Botany, v.78, p.371-381, 1996.

RUSSELE, M.P. et al. Growth analysis based on degree days. Crop Science, v.24, n.1, p.28-32, 1984.

SHAYKEWICH, C.F. An appraisal of cereal crop phenology modeling. Canadian Journal of Plant Science, v.75, n.2, p.329-341, 1995.

SIÉ et al. Rice crop duration and leaf appearence rate in a variable thermal environment. I. Development of an empirically based model. Field Crops Research, v.57, n.1., p.1-13, 1998.

SOSBAI (Sociedade Sul Brasileira de Arroz irrigado). Arroz irrigado: recomendações técnicas para o sul do Brasil. Santa Maria: SOSBAI, 2005. 159p.

STRECK, N.A. et al. Incorporating a chronology response into the prediction of leaf appearance rate in winter wheat. Annals of Botany, v.92, n.2, p.181-190, 2003.
STRECK, N.A. A temperature response function for modeling leaf growth and development of the African violet (Saintpaulia ionantha Wendl.). Ciência Rural, v.34, n.1, p.55-62, 2004.

STRECK, N.A. et al. Estimativa do plastocrono em meloeiro (Cucumis melo L.) cultivado em estufa plástica em diferentes épocas do ano. Ciência Rural, v.35, n.6, p.1275-1280, 2005.

TIVET, F. et al. Leaf blade dimensions of rice (Oryza sativa L. and Oryza glaberrina Stend). Relationship between tillers and the main stem. Annals of Botany, v.88, p.507-511, 2001.

USDA. Rice area, yield and production. Capturado em 23 nov. 2005. Online. Disponível na Internet: http:// www.faz.usda.gov/psd.

WILHELM, W.W.; McMASTER, G.S. Importance of the phyllochron in studying development and growth in grasses. Crop Science, v.35, n.1, p.1-3, 1995

XUE, Q. et al. Predicting leaf appearance in field-grown winter wheat: evaluating linear and non-linear models. Ecological Modelling, v.175, p.261-270, 2004. 